\title{
USO DE LA METODOLOGÍA BRIM (BRIDGE INFORMATION MODELING) COMO HERRAMIENTA PARA LA PLANIFICACIÓN DE LA CONSTRUCCIÓN DE UN PUENTE DE CONCRETO EN COLOMBIA
}

\section{USING THE METHODOLOGY BRIM (BRIDGE INFORMATION MODELING) AS A TOOL FOR PLANNING THE CONSTRUCTION OF A CONCRETE BRIDGE IN COLOMBIA}

\author{
Juan Sebastián Gaitán Cardona', Adriana Gómez Cabrera²
}

Fecha de recepción: 4 de marzo de 2014

Fecha de aprobación: 14 de julio de 2014

Referencia: J.S. Gaitán Cardona, A. Gómez Cabrera. (2014). Uso de la metodología BrIM (Bridge Information Modeling) como herramienta para la planificación de la construcción de un puente de concreto en Colombia. Ciencia e Ingeniería Neogranadina, 24 (2), pp. 145 - 156

\section{RESUMEN}

En este trabajo se presenta la experiencia del uso de metodologías innovadoras paralaplanificación de la construcción de un puente de concreto en Colombia. Fue aplicada la metodología BrIM (Bridge Information Modeling), con el objetivo de mejorar el rendimiento y desarrollo de la planificación constructiva de este tipo de estructura. La elaboración del proyecto se dividió en tres etapas: la construcción del modelo conceptual, la simulación del proceso constructivo, y la cuantificación de materiales y costos. La experiencia demuestra que la elaboración de modelos paramétricos se puede convertir en una herramienta valiosa para la planificación, diseño y construcción de puentes de diferentes tipologías. Sin embargo, es necesario tener bases estructuradas sobre el uso del software y experiencia en la representación y dibujo de los componentes del puente, con el fin de satisfacer las necesidades de la industria constructora, que son mejorar la calidad, reducir costos, dar herramientas para un control adecuado de la construcción y acortar los tiempos de diseño y producción.

Palabras clave: cuantificación de cantidades de obra, modelación de proyectos, BIM, BrIM, modelación de proyectos de construcción, modelación de puentes.

1. Estudiante Ingeniería Civil. Pontificia Universidad Javeriana, Bogotá, Colombia, juan-gaitan@javeriana.edu.co

2. Ingeniero Civil, M.Sc., Profesor investigador, Grupo Investigación Estructuras y Construcción. Pontificia Universidad Javeriana, Bogotá, Colombia, adrianagomez@javeriana.edu.co 


\section{ABSTRACT}

This paper shows the experience of using innovative methodologies for planning the construction of a concrete bridge in Colombia. The BrIM (Bridge information modeling) methodology was applied in order to improve the performance and planning development of the construction of this type of structure. The project development was divided into three stages: the construction of the conceptual model, the simulation of the construction process, and the quantification of materials and costs. The experience showed that the integrated models can become a valuable tool in the process of planning, designing and constructing different types of bridges. In order to use them, however, it is necessary to know the fundamentals of software use and have previous experience in modeling and representing the bridge components. In that case, the needs of the construction industry, such as improving quality, reducing costs, providing the tools for an adequate control of the construction, and reducing the design and production times could be met.

Keywords: modeling projects, BIM, BrIM, modeling construction projects, modeling of bridges

\section{INTRODUCCIÓN}

La falta de planeación general, la insuficiencia en estudios y diseños, y la deficiencia en la elaboración de los presupuestos, entre otros, han sido identificados como factores que afectan la buena marcha de los proyectos de construcción en Colombia [1]. El uso incipiente de tecnología en el sector de la construcción ha hecho que se rezague en avance $y$ eficiencia respecto a otras industrias, pues históricamente se ha considerado lenta para adoptar innovaciones [2].

Tradicionalmente, en la etapa inicial de los proyectos de construcción, los diseños, la cuantificación de materiales, la programación de obra y el presupuesto no se relacionan de manera adecuada y los profesionales trabajan de manera independiente. Esta situación genera problemas en el momento de la ejecución del proyecto como inconsistencias de diseño, ausencia de programación detallada de actividades y planos obsoletos, entre otros, que se manifiestan en pérdidas en tiempo y sobrecostos. La ausencia de un diseño integrado no permite identificar errores o incompatibilidades en etapas iniciales del proyecto, así como aplicar el concepto de «constructability» o «buildability», término implementado para referirse a proyectos de construcción cuyo diseño facilita la fase constructiva [3].

A nivel mundial, las tecnologías de información son cada vez más aplicadas en todos los sectores productivos. Particularmente en el sector de la construcción se están implementando tecnologías de BIM (Building Information Modeling o Modelado de la Información de Construcción), que si son implementadas correctamente, facilitan un diseño y un proceso constructivo más apropiado que resulta en obras civiles de mayor calidad, a un costo más bajo, y en un tiempo más reducido de ejecución [4].

El concepto de BIM es el de modelar como objetos las estructuras de un proyecto 
civil, y darles a estos objetos una serie de características que lo identifiquen y definan para obtener al final modelos paramétricos de edificaciones o proyectos de infraestructura que pueden ser leídos por un sistema computarizado para brindar información automática e integrada. El concepto de BIM transforma el trabajo colaborativo desarrollado por parte de los profesionales involucrados en un proyecto, que tradicionalmente consiste en intercambio de planos y documentos en 2 dimensiones, y que generalmente ha causado problemas de incompatibilidades y de diseño [5].

Según un estudio denominado «The business value of $\mathrm{BIM} \gg[6]$, se dio a conocer el crecimiento del uso de BIM en las empresas de Norteamérica que pasó de un $28 \%$ en el 2007 a un $48 \%$ para el 2009 , lo que implica un crecimiento de un $71 \%$ en dos años. El $45 \%$ de los encuestados lo aprovecha pero sabe que aún hay más y sólo el 3\% trabaja el BIM en toda su capacidad. En Europa, para la misma fecha, 36\% de las empresas dedicadas a la construcción o actividades relacionadas con la industria de la construcción, ubicadas en Europa occidental, reportaron estar implementando la metodología de BIM en sus proyectos.

A partir del BIM, aplicado a edificaciones, se ha desprendido el concepto de BrIM (Bridge Information Modeling o Modelado de la Información de Puentes), aplicado a puentes, que es el objeto central de este trabajo. Éste, por un lado, analiza la viabilidad de dicho concepto a partir de un estudio de caso y, así mismo, se consolida como una herramienta para involucrar a los estudiantes con estas nuevas metodologías, con el objetivo de contar con herramientas adicionales y modernas en el proceso de formación de estudiantes de Ingeniería Civil.
El sector de la construcción en Colombia enfrenta actualmente varios retos. Por un lado, el Reporte Global de Competitividad 2012-2013 ubica a Colombia en el puesto 126 en la calidad de su infraestructura vial, lejos de países como Chile, Ecuador, y México. El país está cercano a países como Guinea y Haití [7]. El gobierno busca contrarrestar el atraso aumentando la construcción de infraestructura, apoyado en el plan de concesiones viales. La importancia de realizar una gestión correcta de la planificación, diseño, construcción y mantenimiento de estos proyectos es evidente.

A nivel internacional se han desarrollado investigaciones para la implementación de nuevas tecnologías que permiten gestionar integralmente los proyectos, en específico los relacionados con la construcción de puentes [8-10]. Finlandia es un país que durante los últimos años ha mantenido indicadores altos de competitividad en temas de infraestructura. Esto se debe a la calidad e innovación que presentan sus investigaciones en sistemas que facilitan la participación, colaboración, comunicación y coordinación de todos los participantes en el análisis de cada etapa del proyecto. Estos sistemas están liderados por un grupo denominado «5D-Bridge» [11], que encabeza parte del desarrollo de metodologías innovadoras debido al trabajo en equipo de compañías privadas, instituciones del estado, consultores, contratistas y fabricantes de software que se ocupan de brindar las pautas para la modelización de puentes y la gestión que se debe realizar durante las etapas del proyecto.

Estas investigaciones dan como resultado metodologías enfocadas directamente en la integración de todas las etapas de construcción de un puente, pasando por el 
diseño y construcción, hasta el mantenimiento y operación de la estructura. Este proceso de generación y gestión de datos durante el ciclo de vida del puente se conoce como BrIM (Bridge Information Modeling). El uso de BrIM se basa en la generación de una representación inteligente de los componentes de la estructura que acogen información detallada y necesaria de todas las etapas del ciclo de vida del puente.

Usando esta metodología, planificar la construcción de la estructura tendría un cambio radical debido a que las herramientas disponibles permiten visualizar en el tiempo la ejecución del proceso constructivo y realizar la estimación de cantidades y costos [1213]. Estos procesos laboriosos que están propensos a errores de apreciación, omisiones e inexactitudes debido a la metodología tradicionalmente empleada pueden minimizarse al hacer uso de un modelo 5D, que se puede utilizar para la planificación, diseño, construcción y operación de la estructura, apoyado en una forma innovadora para el diseño virtual del puente [14].

La elaboración de este proyecto permitió conocer beneficios de la metodología de BrIM para mejorar el rendimiento y desarrollo de la etapa de planificación necesaria para ejecutar la construcción de un puente en Colombia, y cómo esta podría llegar a satisfacer las necesidades de la industria constructora, tales como: mejorar la calidad, reducir costos, contar con nuevas herramientas para un control adecuado de la construcción y acortar los tiempos de diseño y construcción. Se presentan los pasos llevados a cabo para la elaboración del modelo paramétrico, las ventajas y desventajas encontradas, y un análisis de los resultados obtenidos.

\section{METODOLOGÍA}

\subsection{DESCRIPCIÓN DEL PUENTE}

El proyecto corresponde a la construcción de la doble calzada entre la ciudad de Bogotá y Villavicencio. La estructura consiste en un puente recto de vigas simplemente apoyadas con una luz de $20 \mathrm{~m}$, que se requiere para el paso de la vía sobre un cauce existente. La superestructura está conformada por:

- Una losa con un ancho de 12,8 m que sostiene la calzada, dos barreras de tráfico tipo «New Jersey» y un andén peatonal de 1,20 m con su respectiva baranda de seguridad.

- Una calzada de 10,9 m, con un espesor de 0,05 m.

- Cada barrera de tráfico tiene una altura 0,90 m y un ancho de 0,35 m.

- Cuatro vigas rectas postensadas en forma de I, con una altura de 1,15 m, separadas entre ejes una distancia de 3,15 m.

La subestructura está compuesta por dos estribos de concreto con similar configuración, conformados por:

- Un espaldar con un espesor de 0,30 m.

- Una viga cabezal de 1,20 m de altura por 1,80 m de base.

- Cuatro pedestales alineados con cada una de las vigas.

- Dos topes ubicados en la parte externa de las vigas exteriores.

- Dos tapas ubicadas en los extremos del estribo.

- Dos aletas ubicadas a los costados de la estructura, con paredes de 0,35 m y cuya geometría varía dependiendo de las condiciones del terreno.

La cimentación consiste en 6 caissons de concreto de 1,50 m de diámetro que hacen 
parte del apoyo de la viga cabezal, tres en cada estribo.

\subsection{ELABORACIÓN DE MODELO PARAMÉTRICO (3D)}

La modelación de este puente bajo la metodología BriM está representada en el siguiente diagrama de flujo (Figura 1):

El primer paso fue la construcción del modelo en 3 dimensiones a partir del diseño geométrico del puente. A partir del proyecto vial generado y representado en Auto CAD Civil 3D ${ }^{\circledR}$ se obtiene información relevante para el diseño, tal como la ubicación de los elementos estructurales en abscisado, topografía, peraltes de tramo, etc. Se utilizó para esta labor la herramienta Autodesk Revit Structure específicamente la extensión para puentes.

La principal herramienta para generar la representación del puente en un modelo 3D se obtiene a partir de las extensiones que Autodesk ${ }^{\circledR}$ tiene disponible en su plataforma virtual. Esta herramienta permite el modelado de la estructura, el refuerzo, la interoperabilidad y la documentación de toda la construcción del proyecto. Para elaborar este modelo 3D se desarrollaron los pasos que se describen a continuación.

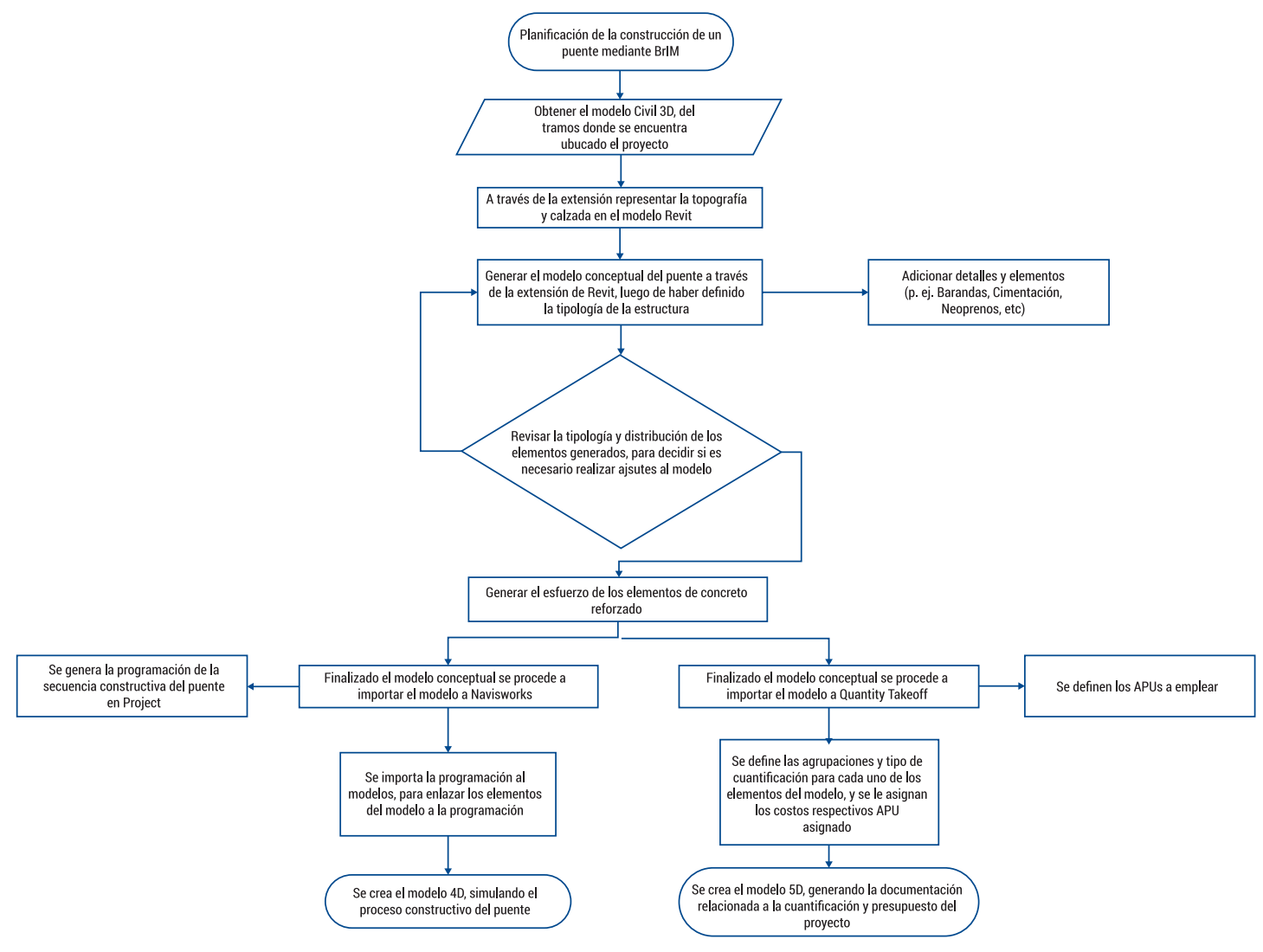

Figura 1. Diagrama de flujo de la metodología utilizada. 
Inicialmente, se realizó la representación del terreno y calzada del proyecto a partir de la integración con el archivo generado en el programa Civil 3D. La extensión permitió extraer las superficies y calzadas presentes en el modelo original.

Para este estudio de caso en particular, se tenía como base para la modelación de la estructura los planos y memorias estructurales, elaboradas de manera tradicional. La modelación inicia con la inserción de cada uno de los elementos, que por defecto en el programa están agrupados por categorías: losas, tableros, estribos, etc. Un ejemplo de esta inclusión de elementos se presenta a continuación para el tablero del puente. Inicialmente se establece el espesor y ancho de la losa en general, pero en las zonas donde se ubican las vigas se incorporan unos parámetros adicionales. Una imagen de esa inclusión se presenta en la Figura 2.

Para cada uno de los elementos incluidos es necesario definir su parametrización: materiales, dimensiones, y también se pueden incluir otras características como proveedor, costo, etc. De esta manera se crearon elementos como calzada, barreras de tráfico, estribos, vigas y pedestales. Un ejemplo de estos elementos puede verse en la Figura 3.

Posteriormente se modeló el estribo a partir de los elementos viga cabezal y espaldar, aletas, apoyo y losa de aproximación. Para la construcción de estos elementos se utilizó una metodología de intersección de diferentes formas para generar los elementos. La visualización final de la superestructura puede verse en la Figura 4.

Luego se realizó la modelación de los caissons de la cimentación. Una imagen de los mismos se puede observar en la Figura 5.

Dentro de esta modelación se incluyó también el refuerzo estructural. En la siguiente tabla se presentan los diámetros y codificación de las barras de refuerzo utilizadas, las cuales fueron creadas con herramientas de dibujo:

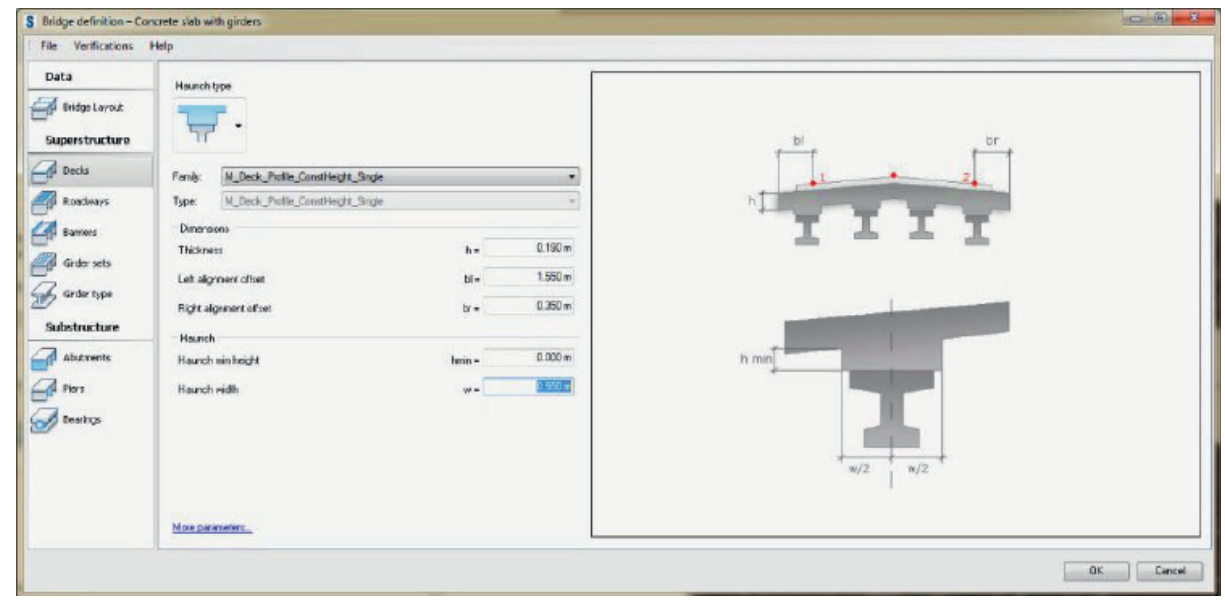

Figura 2. Dimensionamiento de la losa. 


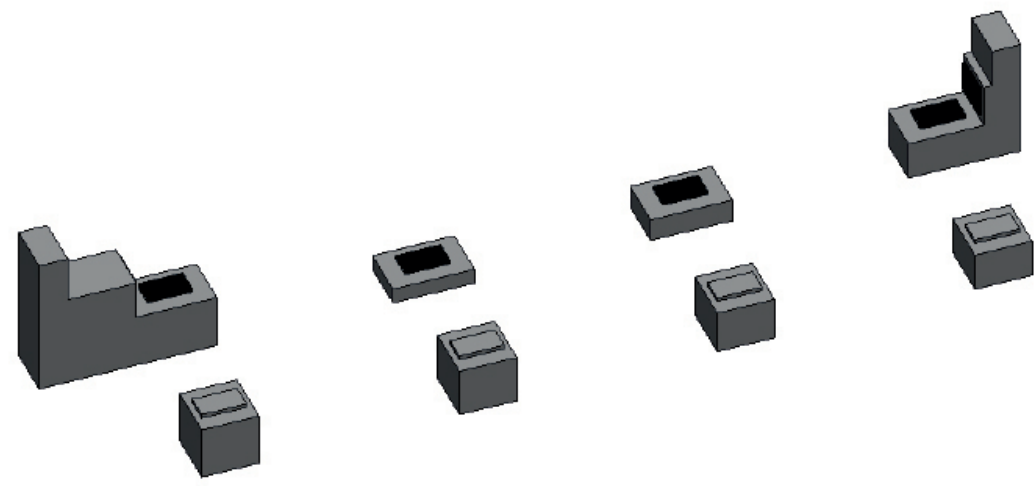

Figura 3. Pedestales modelados.

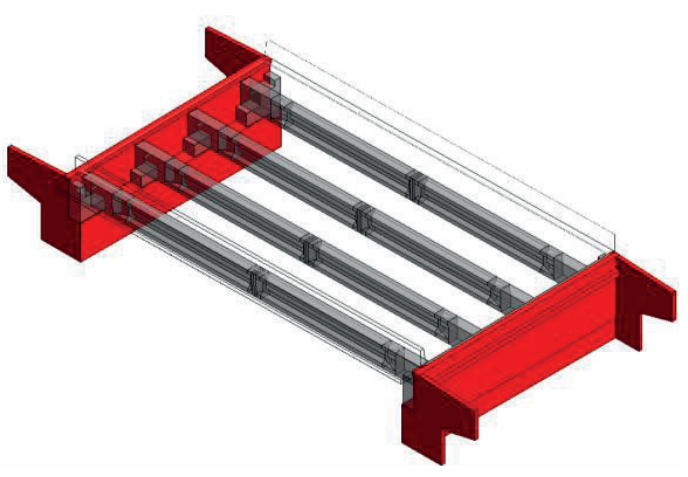

Figura 4. Superestructura.

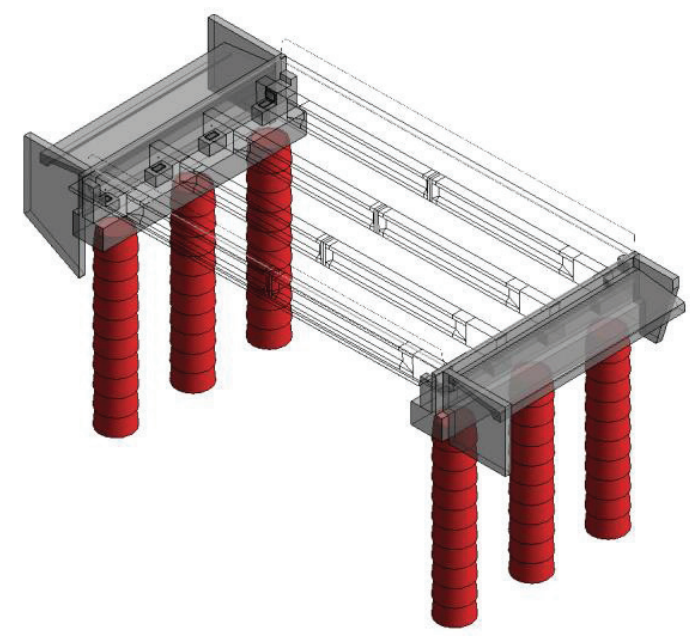

Figura 5. Incorporación de cimentación.

Tabla 1. Longitudes y curvaturas de barras de refuerzo.

\begin{tabular}{|c|c|c|c|c|c|c|c|}
\hline \multirow{3}{*}{ Código } & \multirow{3}{*}{$\begin{array}{l}\text { Diámetro } \\
\text { de } \\
\text { curvatura } \\
(\mathrm{mm})\end{array}$} & \multicolumn{6}{|c|}{ Ganchos } \\
\hline & & \multicolumn{2}{|c|}{$90^{\circ}$} & \multicolumn{2}{|c|}{$135^{\circ}$} & \multicolumn{2}{|c|}{$180^{\circ}$} \\
\hline & & $\begin{array}{c}\text { D. } \\
\text { Curvatura } \\
(\mathrm{mm})\end{array}$ & $\begin{array}{l}\text { Longitud } \\
(\mathrm{mm})\end{array}$ & $\begin{array}{c}\text { D. } \\
\text { Curvatura } \\
(\mathrm{mm})\end{array}$ & $\begin{array}{l}\text { Longitud } \\
(\mathrm{mm})\end{array}$ & $\begin{array}{c}\text { D. } \\
\text { Curvatura } \\
(\mathrm{mm})\end{array}$ & $\begin{array}{l}\text { Longitud } \\
\text { (mm) }\end{array}$ \\
\hline \#3 & 60.0 & 60.0 & 150.0 & 40.0 & 105.0 & 60.0 & 125.0 \\
\hline$\# 4$ & 80.0 & 80.0 & 200.0 & 50.0 & 115.0 & 80.0 & 150.0 \\
\hline$\# 5$ & 95.0 & 95.0 & 250.0 & 65.0 & 140.0 & 95.0 & 175.0 \\
\hline$\# 6$ & 115.0 & 115.0 & 300.0 & 115.0 & 205.0 & 115.0 & 200.0 \\
\hline$\# 7$ & 135.0 & 135.0 & 375.0 & 135.0 & 230.0 & 135.0 & 250.0 \\
\hline$\# 8$ & 155.0 & 155.0 & 425.0 & 155.0 & 270.0 & 155.0 & 275.0 \\
\hline
\end{tabular}




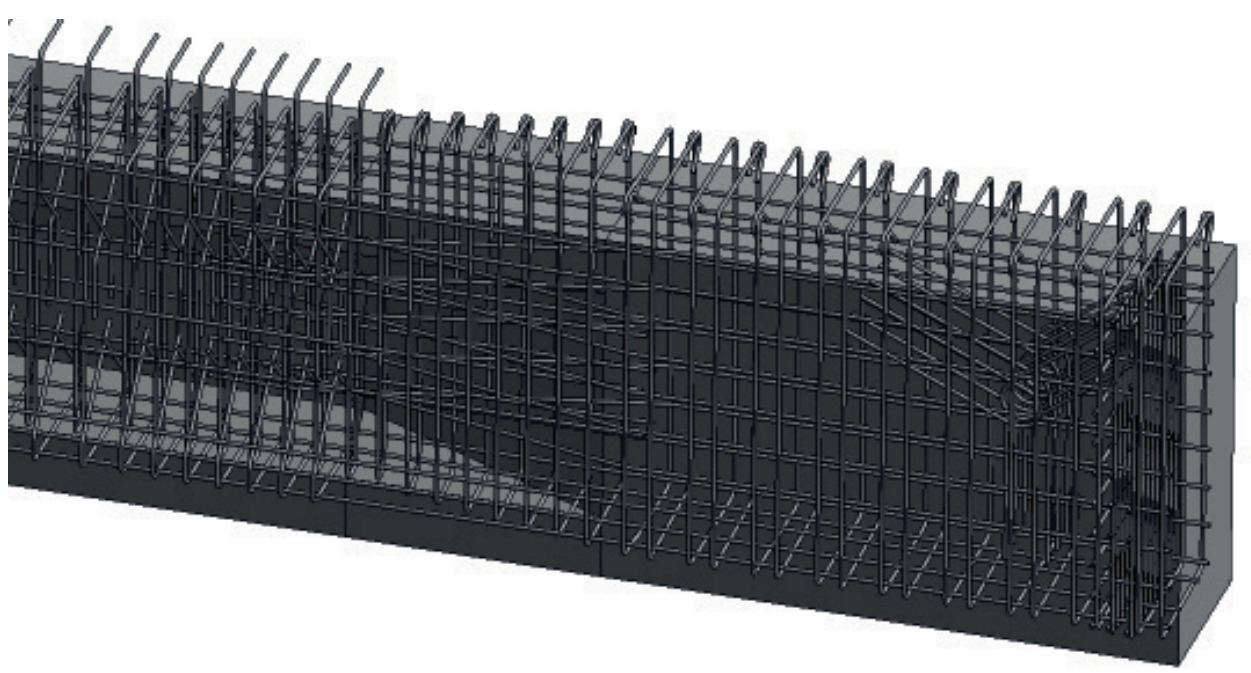

Figura 6. Refuerzo de acero en viga.

La modelación del refuerzo de la estructura se realizó mediante la herramienta de Revit ${ }^{\circledR}$ «Armadura». Una imagen del refuerzo creado en una de las vigas se observa en la Figura 6.

Finalmente se realizó la animación del proceso constructivo en el tiempo, a través de una simulación que representa secuencialmente el orden constructivo de los componentes de la estructura. El primer paso consistió en exportar el modelo Revit ${ }^{\circledR}$ a un formato compatible con Navisworks ${ }^{\circledR}$. Esto se realizó con el fin de poder vincular los componentes a un cronograma. Una imagen de la animación se incluye a continuación. Los caissons en color más oscuro corresponden a los que en el tiempo de la simulación deberían estar construidos (ver Figura 7).

\subsection{CUANTIFICACIÓN DE MATERIALES Y COSTOS (5D)}

Para realizar la cuantificación de cantidades y el cálculo de costos se empleó el programa Autodesk Quantity Takeoff ${ }^{\circledR}$. Se detalla para cada elemento el parámetro deseado: área, volumen, longitud, perímetro, etc. Una visualización del reporte puede observarse a continuación en la Figura 8:

A continuación se presenta una tabla de comparación entre los elementos del puente compuestos de concreto, que permite encontrar la diferencia entre los valores calculados entre lo cuantificado en el modelo BrIM y lo obtenido en su momento de forma manual:

Como puede observarse, las diferencias en cantidades varían. La cuantificación de materiales a través de la metodología BriM es más exacta, pues en la realidad estos ajustes se realizaron en campo generando diferencias en cantidades de obra contractuales.

Una ventaja de esta cuantificación es la facilidad para el análisis del presupuesto, debido a que la estructura organizativa permitía consultar directamente los subtotales de cada uno de los grupos. Además de poder consultar de forma independiente el costo global de cada una de las actividades. 


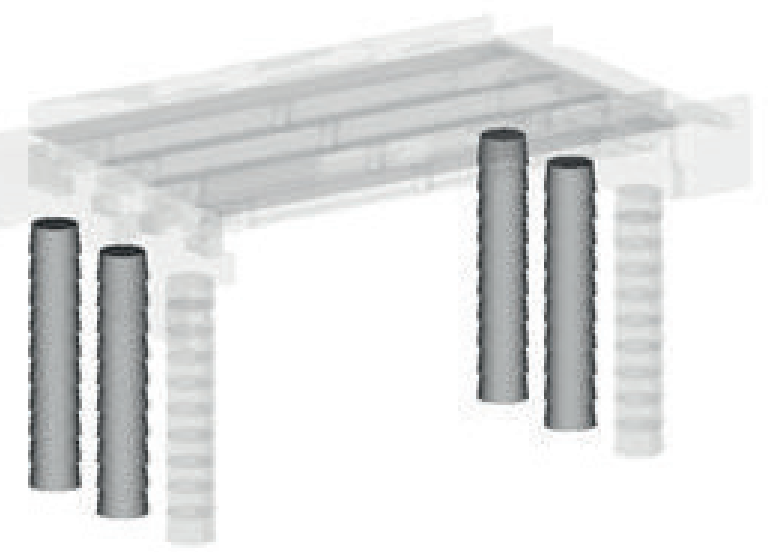

Figura 7. Construcción de los caissons.

\begin{tabular}{|c|c|c|c|c|c|c|c|c|c|c|c|c|}
\hline \multirow[t]{2}{*}{ WBS } & \multirow[t]{2}{*}{ Remarks } & \multirow[t]{2}{*}{ Description } & \multirow[t]{2}{*}{ Quantity 1} & \multicolumn{2}{|c|}{ Equipment Cost } & \multicolumn{2}{|c|}{ Material Cost } & \multicolumn{2}{|c|}{ Subcontractor Cost } & \multicolumn{2}{|c|}{ Labor Cost } & \multirow[t]{2}{*}{ Total Cost } \\
\hline & & & & Unit Cost & Total Cost & Unit cost & Total Cost & Unit cost & Total cost & Unit Cost & Total cost & \\
\hline PTE 2 & & & & & & & & & & & & $\$ 260.962,742.22$ \\
\hline PTE 2. Cimentació & & & & & & & & & & & & 136.054.305.38 \\
\hline \multicolumn{4}{|c|}{ PTE. Cimentación. Caissons profundos } & & & & & & & & & 136.054 .305 .38 \\
\hline $\begin{array}{l}\text { PTE } \\
\text { 2. Cimentación } \\
\text { profundos. Cains } \\
\text { son } 1.50\end{array}$ & 2.2 .010 & Caisson & $156.246 \mathrm{~m}^{3}$ & 175.648 .00 & 27.449.854.37 & 444.246 .00 & 69.411 .488 .83 & 9.915 .00 & 1.549 .175 .26 & 240.927 .00 & 37.643 .786 .93 & 136.054.305.38 \\
\hline
\end{tabular}

Figura 8. Reporte de cantidades de obra.

Tabla 2. Comparación de cuantificaciones de elementos en concreto.

\begin{tabular}{|c|c|c|c|c|c|c|c|}
\hline \multirow{2}{*}{\multicolumn{2}{|c|}{ Elemento }} & \multicolumn{5}{|c|}{ Cantidad } & \multirow{2}{*}{$\begin{array}{c}\% \text { de } \\
\text { Diferencia }\end{array}$} \\
\hline & & \multirow{2}{*}{\begin{tabular}{|l|} 
un \\
$\mathrm{m}^{3}$ \\
\end{tabular}} & \multicolumn{2}{|c|}{ Tradicional } & \multicolumn{2}{|c|}{ Modelo BriM } & \\
\hline \multirow{4}{*}{ Estrbo 1} & $\begin{array}{c}\text { Viga cabezal y } \\
\text { espaldar }\end{array}$ & & 39.10 & \multirow{4}{*}{52.58} & 50.18 & \multirow{4}{*}{53.25} & \multirow{4}{*}{$1.272 \%$} \\
\hline & $\begin{array}{c}\text { Apoyos } \\
\text { (Pedestales, } \\
\text { topes y tapas) }\end{array}$ & $\mathrm{m}^{3}$ & N.A. & & 3.07 & & \\
\hline & Aletas & $\mathrm{m}^{3}$ & 4.54 & & N.A. ${ }^{\star \star}$ & & \\
\hline & $\begin{array}{c}\text { Losa de } \\
\text { aproximación }\end{array}$ & $\mathrm{m}^{3}$ & 8.94 & & N.A.** & & \\
\hline \multirow{4}{*}{ Estrbo 2} & $\begin{array}{c}\begin{array}{c}\text { Viga cabezaly } \\
\text { espaldar }\end{array} \\
\end{array}$ & $\mathrm{m}^{3}$ & 39.10 & \multirow{4}{*}{52.58} & 54.13 & \multirow{4}{*}{57.27} & \multirow{4}{*}{$8.912 \%$} \\
\hline & $\begin{array}{c}\text { Apoyos } \\
\text { (Pedestales, } \\
\text { topes y tapas) }\end{array}$ & $\mathrm{m}^{3}$ & N.A. & & 3.14 & & \\
\hline & Aletas & $\mathrm{m}^{3}$ & 4.54 & & N.A. ${ }^{\star \star}$ & & \\
\hline & $\begin{array}{c}\text { Losa de } \\
\text { aproximación }\end{array}$ & $\mathrm{m}^{3}$ & 8.94 & & N.A.** & & \\
\hline \multicolumn{2}{|c|}{ Cimentación } & $\mathrm{m}^{3}$ & \multicolumn{2}{|c|}{171.93} & \multicolumn{2}{|c|}{156.25} & $9.118 \%$ \\
\hline \multicolumn{2}{|c|}{ Vigas } & $\mathrm{m}^{3}$ & \multicolumn{2}{|c|}{36.30} & \multicolumn{2}{|c|}{37.69} & $3.818 \%$ \\
\hline \multicolumn{2}{|c|}{ Losa } & $\mathrm{m}^{3}$ & \multicolumn{2}{|c|}{62.17} & \multicolumn{2}{|c|}{ N.A } & N.A. \\
\hline \multicolumn{2}{|c|}{ Barrera de trafico } & $m^{3}$ & \multicolumn{2}{|c|}{9.09} & \multicolumn{2}{|c|}{9.85} & $8.394 \%$ \\
\hline
\end{tabular}

*N.A. (No Aplica)

** Cuantificación del elemento incluida en otro objeto 
Por otra parte, algunos Análisis de Precios Unitarios (APU) debieron ser ajustados, a causa de que los tipos de cuantificación asignados presentaban restricciones e impedían relacionar los precios, principalmente las unidades entre lo cuantificado y precio de pago no coincidían. Por ejemplo, inicialmente el APU de «Pilote de concreto fundido in situ, de diámetro 1.50 metros», tenía asignado el metro lineal, pero el modelo no disponía de ese parámetro para su respectiva cuantificación. Por esta razón, se requirió ajustar el APU a la unidad de cubicación $\left(\mathrm{m}^{3}\right)$ con la que sí fue posible la correlación entre cantidad y precio.

Para la cuantificación del acero, el modelo arroja cantidades exactas en longitud y los APU se realizan en kilogramo o tonelada. Fue necesario ajustarlo a metro lineal para poder realizar la simulación de costos.

\section{RESULTADOS Y ANÁLISIS}

La realización de modelos paramétricos para puentes es una herramienta valiosa para la planificación, diseño y construcción. La experiencia adquirida demuestra que esta metodología permite una integración entre diseñadores y constructores que facilita el entendimiento del proyecto, identificar incompatibilidades y minimizar errores.

La principal dificultad quedó evidenciada en la destreza necesaria para el buen uso de las herramientas de los programas que se tienen a disposición. Las plataformas de trabajo tradicionales varían considerablemente con las plataformas de trabajo que están enfocadas en el uso del modelado de información, lo que genera obstáculos para el fácil entendimiento y un libre desarrollo de la creación de objetos y su disposición espacial. Es importante definir la forma de diseñar los elementos, cuantificarlos y determinar sus costos, de acuerdo a la manera en que pueden ser modelados a través de la plataforma.

La creación de un modelo 3D tiene la ventaja de visualizar todos los elementos como un conjunto, lo que permite la exploración del modelo detalladamente a partir de la disposición de gran variedad de vistas. En la metodología tradicional, generalmente se deben extraer fragmentos de información de varios dibujos para generar una visual imaginaria de la estructura completa, lo cual puede generar mayor incertidumbre. La animación en el tiempo también permite entender de una mejor manera el proceso constructivo por parte de quienes desarrollarán esta etapa y se convierte en una herramienta de control, pues es factible cuantificar la ejecución en el momento deseado para simular futuros cortes de obra, de acuerdo con la línea base del proyecto.

Estos modelos paramétricos pueden integrarse con herramientas disponibles para cálculo estructural, análisis de sostenibilidad, análisis de ciclo de vida, entre otros. Esto permite analizar los proyectos desde una nueva perspectiva que contemple las diferentes dimensiones posibles.

\section{CONCLUSIONES}

El modelado de información se puede convertir en una herramienta valiosa para la planificación, diseño y construcción de puentes de diferentes tipologías, pero es necesario tener bases estructuradas sobre el uso del software y experiencia en la representación y 
dibujo de los componentes del puente. Al crear un modelo $3 \mathrm{D}$, se tiene la ventaja de visualizar todos los elementos como un conjunto, permitiendo la exploración del modelo detalladamente a partir de la disposición de gran variedad de vistas, lo cual puede facilitar procesos de «Ingeniería de valor» consistentes en aportar mejoras al proyecto por parte de los profesionales involucrados en su concepción, diseño y construcción.

Una de las ventajas del programa y su uso en el dibujo del refuerzo estructural es la introducción de los parámetros de longitudes de gancho y radios de curvatura. Esto permite visualizar su ubicación en la estructura en 3 dimensiones y la cuantificación se realiza de manera inmediata, lo cual facilita el proceso constructivo. La simulación del proceso constructivo es un recurso valioso para coordinar la interacción de las diferentes actividades ejecutadas por los grupos de trabajo involucrados en la obra, con el propósito de generar la organización íntegra y coordinada de la construcción.

La principal desventaja consiste en que actualmente no se cuenta en Colombia con las familias suficientes que se ajusten a los métodos y elementos tradicionales de diseño. Esto hace que sea necesario crearlos para ir conformando una serie de elementos compatibles con nuestro entorno a nivel de geometría, análisis de precios unitarios, etc.

En este ejercicio académico se realizó el modelo paramétrico a partir de un diseño ya existente para poder identificar ventajas y desventajas y poder comparar con datos reales, pero en la práctica desarrollar el proyecto mediante esta metodología permitirá una mejor concepción, un mejor entendimiento, una disminución de incompatibilidades y un control en obra más preciso.

\section{REFERENCIAS BIBLIOGRAFICAS}

[1] Caicedo, J.M. (2013). Las Obras Públicas: ¿Qué Falla?. Recuperado el 12 de marzo de 1014, de http://www.infraestructura.org. co/noticiasprincipales.php?np_id=330 .

[2] Alarcon, L.F., Fuster, S., Mora, M. \& Sossdorf, D. (2009). Utilización de imágenes y videos digitales para el mejoramiento de la productividad y prevención de riesgos en operaciones de construcción. Encuentro Latinoamericano de economía y Gestión de la Construcción, ELAGEC 2009. Bogotá, Colombia.

[3] Fischer, M. \& Tatum, C.B. (1997). Characteristics of Design-Relevant Constructability Knowledge. Journal of Construction Engineering and Management, 123(3).

[4] Eastman, C.M., Teicholz, P., Sacks, R. \& Liston, K. (2011). BIM Handbook Introduction. En BIM Handbook: A Guide to Building Information Modeling for Owners, Managers, Designers, Engineers, and Contractors. Hoboken, NJ, EE.UU.: John Wiley \& Sons, Inc. doi: 10.1002/9780470261309.ch

[5] Castañeda, C. (2009). Plan para integración del "Building Information Modeling" (BIM) con herramientas de estimación de costos y programación de obra. Encuentro Latinoamericano de Economía y Gestión de la Construcción, ELAGEC 2009. Bogotá, Colombia.

[6] Mc Graw Hill Construction. (2009). The Business Value of BIM: Getting to the 
Bottom Line. Recuperado el 17 de junio de 2014, de http://www.bim.construction. com/research/

[7] World Economic Forum. (2013). Global Competitiveness Report_2013-14. Recuperado el 17 de junio de 2014, http://www3.weforum.org/docs/WEF_ GlobalCompetitivenesReport_201314.p df.

[8] Shin, H.M., Lee, H.M, Oh, S.J \& Chen, J.H. (2011). Analysis and Design of Reinforced Concrete Bridge Column Based on BIM. Procedia Engineering, 14.

[9] Shim, C.S., Yun, N.R. \& Song, H.H. (2011). Application of 3D Bridge Information Modeling to Design and Construction of Bridges. Procedia Engineering, 14.

[10] Halfawy, F.C., Hadipriono, F.C, Duane, J. \& Larew, R. (2005). Development of Model Based Systems for Integrated Design of Highway Bridges. Institute for Research in Construction. International Conference on Civil, Structural and Environmental Engineering Computing, Roma, Italia.

[11] Kivimaki, T. (2010). Bridge Information Modelling (BrIM) and Model Utilization at Worksites in Finland. 27th International Symposium on Automation and Robotics in Construction (ISARC 2010)

[12] Heikkila, M.J. (2005). On The Economy And Benefits Of 3-D Design Method In Bridge Engineering. 22th International Symposium on Automation and Robotics in Construction (ISARC 2005).

[13] Marzouk, M. \& Hisham, M. (2012). Applications of Building Information Modeling in Cost Estimation of Infrastructure Bridges. International Journal of 3-D Information Modeling, 1(2).

[14] Azhar, S. (2011). Building Information Modeling (BIM): Trends, Benefits, Risks, and Challenges for the AEC Industry. Leadership and Management in Engineering, 11(3). 